\title{
Holographic Viscosity of Fundamental Matter
}

\author{
David Mateos, ${ }^{1}$ Robert C. Myers, ${ }^{2,3,4}$ and Rowan M. Thomson ${ }^{3,4}$ \\ ${ }^{1}$ Department of Physics, University of California, Santa Barbara, California 93106-9530, USA \\ ${ }^{2}$ Kavli Institute for Theoretical Physics, University of California, Santa Barbara, California 93106-4030, USA \\ ${ }^{3}$ Perimeter Institute for Theoretical Physics, Waterloo, Ontario N2L 2Y5, Canada \\ ${ }^{4}$ Department of Physics and Astronomy, University of Waterloo, Waterloo, Ontario N2L 3G1, Canada
}

(Received 2 November 2006; published 9 March 2007)

\begin{abstract}
A holographic dual of a finite-temperature $S U\left(N_{c}\right)$ gauge theory with a small number of flavors $N_{f} \ll N_{c}$ typically contains D-branes in a black hole background. By considering the backreaction of the branes, we demonstrate that, to leading order in $N_{f} / N_{c}$, the viscosity to entropy ratio in these theories saturates the conjectured universal bound $\eta / s \geq 1 / 4 \pi$. Given the known results for the entropy density, the contribution of the fundamental matter $\eta_{\text {fund }}$ is therefore enhanced at strong 't Hooft coupling $\lambda$; for example, $\eta_{\text {fund }} \sim \lambda N_{c} N_{f} T^{3}$ in four dimensions. Other transport coefficients are analogously enhanced. These results hold with or without a baryon number chemical potential.
\end{abstract}

DOI: 10.1103/PhysRevLett.98.101601

Introduction. - Recently a proposal was made for a universal bound $\eta / s \geq 1 / 4 \pi$ on the ratio of the shear viscosity to the entropy density of all relativistic quantum field theories [1]. Experimental results from the Relativistic Heavy Ion Collider suggest that, for QCD just above the deconfinement phase transition, $\eta / s$ is close to saturating this bound [2]. Although no theoretical tools with which to calculate transport coefficients in QCD in this regime are available, a large class of gauge theories is accessible to study with the gauge-gravity correspondence [3]. In particular, in the gauge theory limit of large $N_{c}$ and large 't Hooft coupling $\lambda$, the dual description reduces to classical supergravity. Explicit calculations [4,5] and general arguments $[1,6,7]$ have demonstrated that, in this limit, the bound is exactly saturated by a large class of holographic theories. To make contact with real-world QCD, it is clearly important to consider $1 / \lambda$ and $1 / N_{c}$ corrections. For four-dimensional $\mathcal{N}=4$ super Yang-Mills (SYM), the leading $1 / \lambda$ correction raises the value of $\eta / s$ above the bound [8].

A feature common to all of the gauge theories considered in these studies is that the matter transforms in the adjoint representation of the gauge group [9]. Here we study the effect of adding matter in the fundamental representation. In particular, we focus on four-dimensional $S U\left(N_{c}\right)$ SYM coupled to $N_{f}$ fundamental hypermultiplets with $N_{f} \ll N_{c}$. Large- $N_{c}$ counting rules imply that, in the deconfined phase, the contribution of the gluons and adjoint matter to physical quantities is of order $N_{c}^{2}$. Further, the first correction in the absence of fundamental matter is of order 1 ; i.e., it is suppressed by $1 / N_{c}^{2}$. Instead, the relative contribution of fundamental matter is only suppressed by $N_{f} / N_{c}$, and therefore it constitutes the leading correction. This is particularly important in theories for which the bound is exactly saturated at $N_{c}=\infty$, since in these cases whether or not the bound is violated at large but
PACS numbers: 11.25.Tq, 11.10.Wx, 11.15.-q, 25.75.- $\mathrm{q}$

finite $N_{c}$ is completely determined by the leading $1 / N_{c}$ correction.

The dual gravity description is given by $N_{f}$ D7-brane probes [11] in the background of $N_{c}$ D3-branes. At finitetemperature, the latter contains a black hole [12]. Determining modifications of the shear viscosity requires that we go beyond the usual probe approximation and begin to account for the backreaction of the D7-branes. However, to leading order in $N_{f} / N_{c}$, the calculation of $\eta / s$ can be effectively reduced to one in five-dimensional Einstein gravity coupled to a scalar field. General results $[1,6]$ then guarantee that $\eta / s=1 / 4 \pi$. Since the D7-brane contribution to the entropy density is $s_{\text {fund }} \sim \lambda N_{c} N_{f} T^{3}$ [13], the contribution of the fundamental matter to the shear viscosity at strong coupling is enhanced with respect to that dictated solely by large- $N_{c}$ counting rules.

Holographic framework. - The shear viscosity of the gauge theory in the $i j$ plane may be computed via Kubo's formula (with no summation on $i, j$ ):

$$
\eta=\lim _{\omega \rightarrow 0} \frac{1}{2 \omega} \int d t d^{3} x e^{i \omega t}\left\langle\left[T_{i j}(x), T_{i j}(0)\right]\right\rangle .
$$

The stress-energy tensor above is dual on the string side to a metric perturbation $H_{i j}$ polarized along the same twoplane. The retarded correlator can be calculated by taking two functional derivatives of the on-shell string effective action with respect to this perturbation [14]. In the large- $N_{c}$, large- $\lambda$ limit, this action reduces to the type IIB supergravity action coupled to the action of the D7-branes, $I=I_{\mathrm{IIB}}+I_{\mathrm{D} 7}$. Schematically, we have

$$
I=\frac{1}{16 \pi G} \int d^{10} x \sqrt{-g} R-N_{f} T_{\mathrm{D} 7} \int d^{8} x \sqrt{-g_{\text {ind }}}+\cdots,
$$

where $g_{\text {ind }}$ is the induced metric on the D7-branes. The ratio between the normalizations of the two terms above is 
$\varepsilon=16 \pi G N_{f} T_{\mathrm{D} 7}=\lambda N_{f} / 2 \pi N_{c}$, where $\lambda=g_{\mathrm{YM}}^{2} N_{c}$ is the 't Hooft coupling. This ratio controls the relative magnitude of the D7-branes' contribution to physical quantities, e.g., the entropy density [13]. We will assume that $\varepsilon \ll 1$, so that the D7-branes can be treated as a small perturbation; for fixed $\lambda$ this is achieved by taking $N_{f} \ll N_{c}$. We will begin by examining contributions of order $\varepsilon$ in the next section. In the last section, we will comment on the effects of order $\varepsilon^{2}$ and higher.

In the absence of D7-branes, the supergravity background dual to four-dimensional $\mathcal{N}=4 \mathrm{SYM}$ at temperature $T$ is (in the notation of [13])

$$
\begin{gathered}
d s^{2}=d s_{5}^{2}+L^{2} d \Omega_{5}^{2}, \\
d s_{5}^{2}=\frac{(\pi L T \rho)^{2}}{2}\left[-\frac{f^{2}}{\tilde{f}} d t^{2}+\tilde{f} d x_{i}^{2}\right]+\frac{L^{2}}{\rho^{2}} d \rho^{2},
\end{gathered}
$$

where $f(\rho)=1-1 / \rho^{4}, \quad \tilde{f}(\rho)=1+1 / \rho^{4}$, and $L=$ $\left(4 \pi g_{s} N_{c}\right)^{1 / 4} \ell_{s}$ is the asymptotic anti-de Sitter (AdS) radius. There are also $N_{c}$ units of Ramond-Ramond (RR) flux through the five-sphere while the remaining supergravity fields vanish. The metric (3) and (4), possesses an event horizon at $\rho=1$. The entropy density of the gauge theory is then given by the geometric entropy of the horizon [15]

$$
s=\pi^{3} L^{3} T^{3} / 4 G_{5}=\pi^{2} N_{c}^{2} T^{3} / 2,
$$

where $G_{5}=G / \pi^{3} L^{5}$ is the five-dimensional Newton's constant obtained by reduction on the five-sphere.

Now we introduce the D7-branes oriented such that five world volume directions match those of the fivedimensional black hole (4), $y \equiv\left\{t, x^{i}, \rho\right\}$, and the remainder wrap an $S^{3}$ (with a possibly varying radius) inside the $S^{5}$ of (3). We adapt coordinates in this internal space such that $d \Omega_{5}^{2}=d \theta^{2}+\sin ^{2} \theta d \Omega_{3}^{2}+\cos ^{2} \theta d \phi^{2}$ and describe the D7-branes embedding as $\chi=\chi(\rho)$, with $\chi=\cos \theta$. To order $\varepsilon^{0}$, this is determined by extremizing the D7brane action in the background (3) and (4). Asymptotically, one finds $\chi=m / \rho+c / \rho^{3}+\cdots$, where $m$ and $c$ are proportional to the quark mass $M_{q}$ and condensate $\langle\bar{\psi} \psi\rangle$, respectively. In the interior, the D7-branes may or may not reach the black hole horizon $[13,16]$.

Viscous branes. - The calculation of the shear viscosity proceeds as follows: First, one solves the (linearized) equation of motion for a metric perturbation $H$ around the appropriate background. Next, one evaluates the appropriate action for the perturbed background to quadratic order in $H$. A second derivative of the on-shell action then yields the desired two-point function (1) [14].

Without D7-branes, the appropriate action is $I_{\text {IIB }}$ and the background is given by (3). In the presence of the D7branes, the action is supplemented by $I_{\mathrm{D} 7}$. To first order in $\varepsilon$, this affects the calculation in three ways. First, the branes produce $\mathcal{O}(\varepsilon)$ corrections to the metric (3), as well as to the dilaton and the RR axion, since they act as new sources in the field equations arising from the combined action. These background corrections lead to modifications of the field equation satisfied by $H$. Second, the branes also modify the $H$ field equation directly through the extra source terms originating from varying $I_{\mathrm{D} 7}$ with respect to $H$. Third, the second derivative of the on-shell action, which yields the correlator in Kubo's formula, may acquire contributions from $I_{\mathrm{D} 7}$.

Further effects would enter a full calculation of $\eta$ at higher orders in $\varepsilon$. For example, the background corrections modify the brane embedding, but this only begins to contribute to the on-shell action at $\mathcal{O}\left(\varepsilon^{2}\right)$. In fact, we will now show that only the first two of the above possible modifications contribute at $\mathcal{O}(\varepsilon)$. Moreover, the only relevant background correction is the zero-mode (on the fivesphere) of the five-dimensional black hole metric (4).

We begin by considering the third set of possible contributions. Expanding the brane action around the $\mathcal{O}\left(\varepsilon^{0}\right)$ background, one finds that, because $H$ enters the action nonderivatively, the $H^{2}$ terms do not have a form which will contribute in the Kubo formula [14]. However, turning on $H$ also induces a correction $\delta \chi=\mathcal{O}\left(H^{2}\right)$ in the embedding of the branes. This leads to a surface term of the right form in the variation of the D7-brane action:

$$
\left.\delta I_{\mathrm{D} 7} \sim \frac{\partial \mathcal{L}_{\mathrm{D} 7}}{\partial\left(\partial_{\rho} \chi\right)} \delta \chi\right|_{\rho_{\max }} .
$$

However, this contribution is proportional to $\delta m$ [13] and hence vanishes because the variation of the action with respect to $H$ is taken while keeping the quark mass fixed.

Consider now corrections to the background (3). While there are $\mathcal{O}(\varepsilon)$ corrections to the dilaton and the RR axion, these only produce $\mathcal{O}\left(\varepsilon^{2}\right)$ contributions to the $H$ field equation because they enter the supergravity action quadratically (in the Einstein frame). We are thus left to consider the contributions of corrections to the spacetime metric. To order $\varepsilon$, the background metric is

$$
g=g_{0}+\varepsilon g_{1}^{(0)}+\varepsilon \sum_{\ell \neq 0} g_{1}^{(\ell)},
$$

where the corrections $g_{1}^{(\ell)}$ are organized by their properties under the $S O(6)$ rotations on $S^{5}$, with $\ell=0$ denoting the invariant contributions [17]. Since the D7-branes only fill an $S^{3}$ in the internal space, they also source the $g_{1}^{(\ell)}$ corrections with $\ell \neq 0$. For the following, an important point is that the functions $g_{1}^{(0)}$ and $g_{1}^{(\ell)}$ respect the symmetries of the background geometry (3) and the brane embedding, i.e., translations in $\left\{t, x^{i}\right\}, S O(3)$ rotations in $x^{i}$, and $S O(4)$ rotations in the internal $S^{3}$. The perturbation $H$ has a similar decomposition:

$$
H=H_{0}+\varepsilon H_{1}^{(0)}+\varepsilon \sum_{\ell \neq 0} H_{1}^{(\ell)} .
$$

Implicitly, $H_{0}$ is an $S O(6)$ singlet and in the absence of D7- 
branes, it is consistent to restrict the perturbation to this sector [4,5]. In the presence of the D7-branes, nontrivial $H_{1}^{(\ell)}$ are sourced when $H_{0}$ is turned on [18]. Indeed, after integration over the $S^{5}$, the supergravity action produces couplings of the form $\varepsilon^{2} \int d^{5} y H_{0} H_{1}^{(\ell)} g_{1}^{(\ell)}$. Similarly, the D7-branes action produces couplings like $\varepsilon^{2} \int d^{5} y H_{0} H_{1}^{(\ell)}$ for modes that are constant on the $S^{3}$. However, as indicated, both types of terms are $\mathcal{O}\left(\varepsilon^{2}\right)$ and so we may neglect their contribution here.

We therefore conclude that, to order $\varepsilon$, we need only consider the zero modes $g_{1}^{(0)}(y)$ and $H_{1}^{(0)}(y)$. Hence the evaluation of the viscosity actually reduces to a fivedimensional calculation. We can make the latter concrete by dimensionally reducing the action $I_{\mathrm{IIB}}+I_{\mathrm{D} 7}$ to five dimensions ignoring all the Kaluza-Klein modes on the five sphere, as well as the other supergravity fields. The five-dimensional action can be written as

$$
\begin{aligned}
I_{5}= & \frac{1}{16 \pi G_{5}} \int d^{5} y \sqrt{-g}\left[R+\frac{12}{L^{2}}-\frac{2 \varepsilon}{\pi L^{2}}\left(1-\chi^{2}\right)\right. \\
& \left.\times \sqrt{1-\chi^{2}+L^{2} g^{\rho \rho}\left(\partial_{\rho} \chi\right)^{2}}\right] .
\end{aligned}
$$

We have only allowed scalar field configurations depending on the radial coordinate, since this suffices for our purposes. This system is just five-dimensional Einstein gravity coupled to a cosmological constant and a(n unusual) scalar field $\chi$. In an $\varepsilon$ expansion, the black hole solutions generated by this auxiliary theory will match the asymptotically AdS part of the original ten-dimensional solution to order $\varepsilon$, i.e., the brane profile $\chi(\rho)$ and the background metric (4) plus the $\mathcal{O}(\varepsilon)$ correction $g_{1}^{(0)}(\rho)$.

The viscosity may now be obtained by calculating the perturbation $H_{i j}$ around the five-dimensional solution and taking the second functional derivative of the action (9) evaluated on-shell. However, the black brane solutions of our auxiliary five-dimensional system satisfy the symmetries required in [1], namely, translational and rotational invariance in the $x^{i}$ directions, and hence the result is guaranteed to satisfy $\eta / s=1 / 4 \pi$. We thus conclude that this universal bound is still saturated in the full tendimensional string theory when working to first order in $\varepsilon$. With the known results for $s_{\text {fund }}$ [13], we have

$$
\eta=\frac{\pi}{8} N_{c}^{2} T^{3}\left[1+\frac{\lambda}{8 \pi^{2}} \frac{N_{f}}{N_{c}} h\left(\frac{\lambda T}{M_{q}}\right)+\cdots\right],
$$

where $h(x)$ satisfies $h(0)=0, h(\infty)=1$, and makes a crossover between both values around $x \sim 1$; for details, see [13]. Therefore the leading correction is enhanced by a factor of $\lambda$ at strong coupling with respect to the $\mathcal{O}\left(N_{f} / N_{c}\right)$ value expected by large- $N_{c}$ counting rules.

The calculation of $s_{\text {fund }}$ in [13] was performed by identifying the Euclidean action of the D7-branes with $F_{\text {fund }} / T$, where $F_{\text {fund }}$ is the free energy contribution of the fundamental matter. The entropy is then determined as $s_{\text {fund }}=$ $-\partial F_{\text {fund }} / \partial T$. This should coincide with the change in the horizon area induced by the presence of the branes. The latter can be explicitly verified for the case of massless quarks, for which $\chi=0$ and $s_{\text {fund }}=\lambda N_{c} N_{f} T^{3} / 16$. We see in (9) that the net effect of these "equatorial" branes is to shift the effective cosmological constant. The corresponding black hole solution is still given by (4), with the replacement $L^{2} \rightarrow L^{2} /(1-\varepsilon / 6 \pi)$. The same replacement in (5) shifts the entropy to order $\varepsilon$ by $\delta s=\lambda N_{c} N_{f} T^{3} / 16$, in perfect agreement with the previous result.

Discussion. - We have reduced the calculation of the contribution of fundamental matter to the shear viscosity to one in five dimensions. An analogous simplification takes place for other transport coefficients that can be extracted from correlators involving local operators with no $R$ charge, since these are dual to modes with no angular momentum on the $S^{5}$. Examples include the speed of sound and the bulk viscosity. Other coefficients that involve $R$-charged operators, such as the $R$-charge diffusion constant [5], or extended strings, such as the jet quenching parameter $\hat{q}$ (see, e.g., [19]), may require a ten-dimensional calculation. Generically, however, we expect the relative contribution of the fundamental matter to be of order $\varepsilon \sim$ $\lambda N_{f} / N_{c}$.

Above, our discussion focused on the D3/D7 system, but the arguments are easily extended to a $\mathrm{D} p / \mathrm{D} q$ system intersecting over $d$ common directions. This is dual to a SYM theory in $p+1$ dimensions coupled to fundamental matter confined to a $(d+1)$-dimensional defect, which breaks translational invariance along the $p$ - $d$ orthogonal directions. To calculate the viscosity along the translationally invariant directions, the simplest approach is to compactify these extra directions. The previous arguments go through essentially unchanged except for the fact that the index $\ell$ now labels momentum modes both along the $S^{8-p}$ transverse to the $\mathrm{D} p$-branes and along the $p$ - $d$ directions orthogonal to the defect. In this case the calculation of the leading contribution of the fundamental matter to the $\eta / s$ ratio can be reduced to one in $(d+2)$-dimensional Einstein gravity coupled to a set of scalar fields. In addition to the scalar $\chi$ above, this set now includes the dilaton and the metric components governing the size of the internal $S^{8-p}$ of the background geometry and the size of the ( $p-$ d)-dimensional space orthogonal to the defect. Further, the form of the $(d+2)$-dimensional gravity theory and the background guarantees that $\eta / s=1 / 4 \pi$. The leading result for the entropy density was determined in [13] and hence we have $\eta_{\text {fund }} \sim N_{c} N_{f} T^{d} g_{\text {eff }}(T)^{2(d-1) / 5-p}$, where $g_{\text {eff }}^{2}(T)=\lambda T^{p-3}$ is the dimensionless effective 't Hooft coupling for a $(p+1)$-dimensional theory at temperature $T$ [20]. Here the gauge-gravity duality is only valid in the strongly coupled regime [20] and hence we again see an enhancement beyond the large- $N_{c}$ counting. The same line of argument can also be implemented for the $\mathrm{D} p / \mathrm{D} q / \mathrm{D} \bar{q}$ systems [21]. 
The $U\left(N_{f}\right) \simeq S U\left(N_{f}\right) \times U(1)_{B}$ gauge symmetry on the $\mathrm{D} q$-branes is a global, flavor symmetry of the dual gauge theory. The results above also hold when a baryon number chemical potential for the $U(1)_{B}$ charge is introduced. This is dual [22] to turning on the time component of the gauge potential on the $\mathrm{D} q$-branes, $A_{0}(\rho)$ [23]. The arguments from above go through essentially unchanged, except that an additional vector $A_{\mu}$ is added to the reduced $(d+2)$-dimensional Einstein gravity theory [7].

Above we have worked to the lowest order in the parameter $\varepsilon \sim \lambda N_{f} / N_{c}$ that controls the backreaction of the D7-branes on the D3-brane geometry. We have argued that to this order one may ignore all effects of this backreaction except for those on the noncompact part of the metric. We regard the agreement between the entropy density as calculated in [13] and as obtained here from the change in the horizon area as a quantitative consistency check of this approach. In calculating beyond order $\varepsilon$, the internal modes, e.g., $g_{1}^{(\ell)}(y)$, and other supergravity fields, will all play a role. Further at $\mathcal{O}\left(\varepsilon^{2}\right)$, quantum effects will have to be considered [24]. Closed string loop corrections are an expansion in $g_{s}^{2} \sim \lambda^{2} / N_{c}^{2}$. Thus if as above $N_{f}$ is fixed, the loop corrections may be of the same magnitude as the higher order D7-brane contributions, i.e., $g_{s}^{2} \sim \varepsilon^{2}$ if $N_{f}=\mathcal{O}(1)$.

The holographic field theories with fundamental matter are naturally organized according to three expansions in $1 / \lambda, 1 / N_{c}$, and $N_{f} / N_{c}$. To suppress string loop corrections with respect to the backreaction of the branes one must keep $N_{f} / N_{c}$ fixed while taking $N_{c} \rightarrow \infty$. The resulting classical solution would resum the effects of the fundamental matter in the $N_{f} / N_{c}$ expansion, keeping the leading order contributions in $1 / \lambda$ and $1 / N_{c}$. A fully backreacted solution is singular for the D3/D7 system [25], since the dual gauge theory possesses a Landau pole at some (finite) high-energy scale $\Lambda_{\mathrm{UV}}$, but this should not affect the long wavelength, hydrodynamic behavior. Similarly, in the case of a general $\mathrm{D} p$-brane background (with $p \neq 3$ ), one finds strong coupling or curvature divergences in the far UV. It may be possible to prove that the bound is still saturated in these solutions by extending the arguments of [6,7].

We thank H. Elvang, R. Emparan, S. Mathur, and S. Shenker for discussions, A. Starinets for collaborating at an early stage, and A. Buchel for correspondence. D. M. thanks the ACP and the KITP for hospitality. Research at PI is supported in part by the Government of Canada through NSERC and by the Province of Ontario through MRI. We also acknowledge support from NSF Grant No. PHY-0244764 (D. M.), an NSERC Discovery grant (R.C.M.) and the NSERC Canada Graduate program (R. M. T.). Research at the KITP was supported in part by the NSF under Grant No. PHY99-07949.
[1] P. Kovtun et al., J. High Energy Phys. 10 (2003) 064; Phys. Rev. Lett. 94, 111601 (2005).

[2] E. Shuryak, Prog. Part. Nucl. Phys. 53, 273 (2004); D. Teaney, Phys. Rev. C 68, 034913 (2003).

[3] J. M. Maldacena, Adv. Theor. Math. Phys. 2, 231 (1998); Int. J. Theor. Phys. 38, 1113 (1999).

[4] G. Policastro et al., Phys. Rev. Lett. 87, 081601 (2001).

[5] G. Policastro et al., J. High Energy Phys. 09 (2002) 043.

[6] A. Buchel and J. T. Liu, Phys. Rev. Lett. 93, 090602 (2004); A. Buchel, Phys. Lett. B 609, 392 (2005).

[7] P. Benincasa, A. Buchel, and R. Naryshkin, Phys. Lett. B 645, 309 (2007).

[8] A. Buchel et al., Nucl. Phys. B707, 56 (2005); P. Benincasa and A. Buchel, J. High Energy Phys. 01 (2006) 103.

[9] An exception is Ref. [10]. However, the temperature is not a free parameter.

[10] R. Casero et al., Phys. Rev. D 73, 086005 (2006).

[11] A. Karch and E. Katz, J. High Energy Phys. 06 (2002) 043.

[12] E. Witten, Adv. Theor. Math. Phys. 2, 505 (1998).

[13] D. Mateos, R. C. Myers, and R. M. Thomson, Phys. Rev. Lett. 97, 091601 (2006); hep-th/0701132.

[14] D. T. Son and A. O. Starinets, J. High Energy Phys. 09 (2002) 042; C. P. Herzog and D. T. Son, J. High Energy Phys. 03 (2003) 046.

[15] S. S. Gubser et al., Phys. Rev. D 54, 3915 (1996).

[16] J. Babington et al., Phys. Rev. D 69, 066007 (2004); M. Kruczenski et al., J. High Energy Phys. 05 (2004) 041.

[17] As the metric is a tensor, invariant means $g_{1}^{(0)}$ has vanishing Lie derivatives under the $S O(6)$ Killing symmetries of the background (3). Hence as well as perturbations of the AdS black hole (4), $g_{1}^{(0)}$ includes perturbations in the size of the five sphere. However, this scalar is removed from the $O(\varepsilon)$ calculation by going to the Einstein frame in the reduced action (9).

[18] With D7-branes, $H_{0}$ also sources other supergravity fields at $O\left(\varepsilon H_{0}^{2}\right)$. However, these perturbations do not contribute to the shear viscosity at $O(\varepsilon)$.

[19] C. P. Herzog et al., J. High Energy Phys. 07 (2006) 013; H. Liu, K. Rajagopal, and U. A. Wiedemann, Phys. Rev. Lett. 97, 182301 (2006); S. S. Gubser, Phys. Rev. D 74, 126005 (2006); J. Casalderrey-Solana and D. Teaney, Phys. Rev. D 74, 085012 (2006).

[20] N. Itzhaki et al., Phys. Rev. D 58, 046004 (1998).

[21] T. Sakai and S. Sugimoto, Prog. Theor. Phys. 113, 843 (2005); E. Antonyan et al., hep-th/0608177.

[22] R. Apreda et al., Phys. Rev. D 71, 126002 (2005); N. Horigome and Y. Tanii, J. High Energy Phys. 01 (2007) 072; S. Kobayashi et al., J. High Energy Phys. 02 (2007) 016.

[23] This baryon number chemical potential should not be confused with the $R$-charge chemical potential dual to angular momentum on the sphere.

[24] We expect Hawking radiation to be of order $N_{f}^{2} / N_{c}^{2}$, i.e., suppressed by $1 / \lambda^{2}$ relative to $\varepsilon^{2}$.

[25] I. Kirsch and D. Vaman, Phys. Rev. D 72, 026007 (2005); B. A. Burrington et al., J. High Energy Phys. 02 (2005) 022 . 